\title{
Funcionalidade da família dos pacientes com doença pulmonar obstrutiva crônica
}

\author{
Functionality of family of the patients with chronic obstructive lung disease \\ Funcionalidad de la familia de pacientes con enfermedad pulmonar obstructiva crónica \\ Carolina Schmitt 1 , Sabrina Antonio de Souza르. Camila da Silva Brinques ${ }^{3}$, Thaís Franco da Silva ${ }^{4}$, \\ Andréa Lúcia Gonçalves da Silva ${ }^{5}$, Renata Trimer
}

RESUMO I A doença pulmonar obstrutiva crônica (DPOC) é altamente limitante, tanto nos aspectos físicos, quanto socioemocionais, causando dificuldades significativas no cotidiano dos pacientes. Além de afetar os pacientes, a DPOC também influência na vida dos familiares e amigos próximos, pois deles vem a rede de apoio de que os pacientes necessitam. O objetivo deste artigo é avaliar a funcionalidade da família no cotidiano de pacientes acometidos por DPOC. É um estudo transversal que avaliou a funcionalidade de família de pacientes com DPOC em diferentes estágios da doença e que realizam Programa Reabilitação Cardiorrespiratória (PRC). Utilizou-se o instrumento APGAR de Família, sendo este, um acrônimo caracterizado por $\mathrm{A}=$ (Adaptation/ Adaptação), $\mathrm{P}=$ (Partnership/Companheirismo), $G=$ (Grow/Desenvolvimento), $A=$ (Affection/Afetividade), $\mathrm{R}=$ (Reso/ve/Capacidade resolutiva), composto por 5 questões avaliadas em "sempre", "algumas vezes” " "nunca”, o que somados resultam em "elevada disfunção familiar", moderada disfunção familiar" e "boa funcionalidade familiar". Foram avaliados 21 pacientes DPOC [sexo masculino (n=11; 52,3\%); idade média 66,3ะ10 anos], com estadiamento entre moderado a muito severo. Através do APGAR da Família identificamos 2 pacientes com elevada disfunção familiar; 2 pacientes com moderada disfunção familiar; 17 pacientes com boa funcionalidade familiar. Dos que relataram elevada e moderada disfunção familiar, os acrômios mais comprometidos foram "companheirismo", "afetividade" e "desenvolvimentos". O grau de obstrução das vias áreas associou-se moderada e positivamente com a funcionalidade da família ( $r=0,697 ; p=0,004)$. Pacientes com DPOC participantes do PRC apresentaram boa funcionalidade familiar no seu cotidiano e a gravidade da doença esteve associada a esta funcionalidade. Pacientes que apresentaram maior disfunção familiar relataram falta de "companheirismo", "afetividade" e "desenvolvimentos". Descritores | Isolamento Social; Relações Familiares; Doença Pulmonar Obstrutiva Crônica ; Afeto; Adaptação Psicológica.

ABSTRACT I Background: Chronic Obstructive Pulmonary Disease (COPD) is highly limiting, both physically and socioemotionally, causing significant difficulties in the daily lives of patients. In addition, COPD also influences the lives of family and friends, because theirs who come to the support network that patient need. Objective: To evaluate the family functionality in the daily life of COPD patients. Methods: This cross-sectional study evaluated the family functionality in COPD patients with different stages of disease and underwent Cardiorespiratory Rehabilitation Program (CRP). The Family APGAR instrument was used and it is an acronym characterized by $A=$ (Adaptation / Adaptation), P = (Partnership / Companionship), G = (Grow / Development), A = (Affection / Affectivity), $\mathrm{R}=$ (Resolve / Resolving capacity), composed of 5 questions assessed in "always", "sometimes" and "never", which together result in "high family dysfunction", moderate family dysfunction "and" good family functionality”. We evaluated 21 COPD patients

\footnotetext{
'Graduação em Fisioterapia, Universidade de Santa Cruz do Sul, Santa Cruz do Sul, RS, Brasil. email: ORCID:0000-0002-9263-2368 ${ }^{2}$ Graduação em Fisioterapia, Universidade de Santa Cruz do Sul, Santa Cruz do Sul, RS, Brasil. ORCID:0000-0002-4708-9028 ${ }^{3}$ Graduação em Fisioterapia, Universidade de Santa Cruz do Sul, Santa Cruz do Sul, RS, Brasil. ORCID: https:0000-0002-7067-7354 ${ }^{4}$ Graduação em Fisioterapia, Universidade de Santa Cruz do Sul, Santa Cruz do Sul, RS, Brasil. ORCID:/0000-0002-9823-8601 ${ }^{5}$ Docente do Curso de Fisioterapia, Departamento de Educação Física e Saúde, Universidade de Santa Cruz do Sul, RS, Brasil. ORCID: 0000-0002-8893-286X

${ }^{6}$ Docente do Curso de Fisioterapia, Departamento de Educação Física e Saúde, Universidade de Santa Cruz do Sul, RS, Brasil. ORCID:/0000-0002-9635-1694
} 
[male gender ( $n=11 ; 52.3 \%$ ); mean age $66.3 \pm 10$ years], status disease between moderate to very severe. Through of the Family APGAR we identified 2 patients with high family dysfunction; 2 patients with moderate family dysfunction; 17 patients with good family functionality. Among those who reported high and moderate family dysfunction, the most compromised acromia's were "companionship", "affection" and "developments". The degree of airway obstruction was moderately and positively associated with family functionality $(r=0.697 ; p=0.004)$. Patients with COPD who participated in CRP had good family functionality in their daily lives and the severity of the disease was associated with this functionality. Patients with high family dysfunction reported lack of "companionship", "affection" and "developments".

Keywords | Social Isolation; Family Relations; Pulmonary Disease, Chronic Obstructive; Affect; Adaptation Psychological.

RESUMEN | La enfermedad pulmonar obstructiva crónica (EPOC) es muy limitante, tanto en aspectos físicos como socioemocionales, causando dificultades significativas en la vida diaria de los pacientes. Además de afectar a los pacientes, la EPOC también influye en la vida de los familiares y amigos cercanos, ya que de ellos proviene la red de apoyo que los pacientes necesitan. El objetivo de este artículo es evaluar la funcionalidad de la familia en la vida cotidiana de los pacientes afectados por EPOC. Se trata de un estudio transversal que evaluó la funcionalidad familiar de pacientes con EPOC en diferentes etapas de la enfermedad y que realizan un Programa de Rehabilitación Cardiaca (PRC). Se utilizó el instrumento APGAR familiar, que es un acrónimo caracterizado por $\mathrm{A}=$ (Adaptation/Adaptación), $\mathrm{P}=$ (Partnership/Compañerismo), G = (Grow/Desarrollo), A = (Affection) Afectividad), $\mathrm{R}=$ (Reso/ve/Capacidad de resolución), compuesto por 5 preguntas evaluadas en "siempre", "a veces" y "nunca", que combinadas resultan en "disfunción familiar severa", "disfunción familiar moderada" y "buena funcionalidad familiar". Se evaluaron 21 pacientes con EPOC [sexo masculino ( $n=11 ; 52,3 \%$ ); edad media $66,3 \pm 10$ años], con una estadificación que varía de moderada a muy grave. A través del APGAR familiar identificamos 2 pacientes con severa disfunción familiar; 2 pacientes con disfunción familiar moderada; 17 pacientes con buena funcionalidad familiar. De los que relataron disfunción familiar severa y moderada, los acrónimos más comprometidos fueron “compañerismo", "afectividad” y "desarrollo”. El grado de obstrucción de las vías se asoció moderada y positivamente con la funcionalidad de la familia ( $r=0,697 ; p=0,004)$. Los pacientes con EPOC que participaron en el PCR presentaron una buena funcionalidad familiar en su vida diaria y la gravedad de la enfermedad se asoció a esta funcionalidad. Los pacientes que presentaron mayor disfunción familiar relataron falta de "compañerismo", "afectividad" y "desarrollo". Palabras clave | Aislamiento social; Relaciones Familiares; Enfermedad Pulmonar Obstructiva Crónica ; Afecto; Adaptación Psicológica.

\section{INTRODUÇÃO}

Há um interesse crescente no papel das afecções psicológicas em pacientes com doenças crônicas pelo seu impacto na morbidade e mortalidade dos sujeitos ${ }^{1}$. Estudos sugerem que os distúrbios emocionais, tais como depressão e ansiedade, são comuns entre pacientes com DPOC, pois têm um efeito prejudicial sobre a qualidade de vida, afetando o funcionamento emocional, social e físico ${ }^{1,2}$. Entre as doenças psiquiátricas mais comumente encontradas nos pacientes com DPOC podemos destacar os transtornos depressivos, de ansiedade e as crises de pânico, bem como a ideação suicida ${ }^{2}$.

A doença pulmonar obstrutiva crônica (DPOC) é uma patologia frequente e limitante, tanto em aspectos físicos, quanto psicossociais, por causar dificuldades significativa no cotidiano, levando ao isolamento social e afetando a qualidade de vida ${ }^{3}$. Em decorrência destas alterações, pacientes com DPOC sofrem de carência da liberdade e, consequentemente, aqueles que os cuidam e/ou estão ao seu redor servindo como rede de apoio também sofrem.
Segundo os dados do boletim epidemiológico do Ministério da Saúde ${ }^{4}$, as doenças respiratórias crônicas (DRC) representam cerca de $7 \%$ da mortalidade global, o que corresponde a 4,2 milhões de óbitos anuais. No Brasil, em 2011, as DRC foram a terceira causa de morte no conjunto de doenças crônicas não transmissíveis (DCNT). A DPOC tem sua eclosão prevista para o ano de 2020, em que se estima que será a terceira maior causadora de morte no mundo.

Os transtornos psiquiátricos em paciente com DPOC são de ordem multifatorial, podendo estar relacionados com o tabagismo, a dispneia, o medo, a dor, a hipoxemia, a limitação às atividades de vida diária e com internações hospitalares frequentes ${ }^{2}$. Todos estes fatores associados aos sintomas conduzem ao sentimento de incompetência física que agrava o quadro depressivo ${ }^{5}$.

Assim, considerando a gravidade da doença e todas as limitações físicas, sociais e mentais destes pacientes, associada com a necessidade de uma rede de apoio para auxiliar nas atividades de seu cotidiano, o presente estudo teve como objetivo avaliar a funcionalidade da família no cotidiano de pacientes acometidos por DPOC. 


\section{METODOLOGIA}

Estudo transversal, do tipo estudo de casos, com amostragem de conveniência, avaliou pacientes com diagnóstico de DPOC participantes de um programa de reabilitação cardiorrespiratória (PRC). Foram incluídos no estudo pacientes adultos, com bons aspectos cognitivos, de ambos os sexos, independente do estado civil e que consentiram e assinaram o termo de consentimento livre e esclarecido. Foram excluídos os pacientes com desordens musculoesqueléticas e/ou sequelas neurológicas, doença metabólica não controlada e diagnóstico clínico confirmado de câncer de pulmão.

\section{Procedimentos}

Todos os pacientes foram avaliados para o levantamento de dados clínicos e sociodemográficos, como: idade, sexo, etnia, índice de massa corporal (IMC), histórico de tabagismo, consumo de medicamentos antidepressivos e ansiolíticos, escolaridade e grau de parentesco que os pacientes recorrem em situações de necessidade pessoal. Foram coletados dados espirométricos através do espirômetro digital Microloop ${ }^{\mathrm{TM}}$ Spirometer, identificandose o volume expiratório no primeiro segundo $\left(\mathrm{VEF}_{1}\right)$, a capacidade vital forçada (CVF) e a relação $V_{E F} / C V F$, através da realização de três medidas de acordo com Diretrizes da American Thoracic Society (ATS) ${ }^{6}$ e a curva que apresentasse o melhor desempenho foi comparada com os valores preditos na literatura e descritos em porcentagem do predito ${ }^{7}$. Para estratificação do estadiamento da doença foi seguida as Diretrizes Internacionais do Global Initiative for Chronic Obstructive Lung Disease ${ }^{8}$.

O instrumento APGAR de Família trata-se de um acrônimo caracterizado por: $\mathrm{A}=$ Adaptation (Adaptação), que representa a satisfação do membro familiar com a assistência recebida quando recursos familiares são necessários; $\mathrm{P}=$ Partnership (Companheirismo), compreendido como a satisfação do membro familiar com a reciprocidade nas comunicações familiares e na solução de problemas; $\mathrm{G}=$ Grow (Desenvolvimento), que representa a satisfação do membro familiar com a liberdade disponibilizada pela família para mudanças de papéis para alcance de maturidade ou desenvolvimento emocional; $\mathrm{A}$ = Affection (Afetividade), que indica a satisfação do membro familiar com a intimidade $\mathrm{e}$ as interações emocionais em seu contexto familiar; $\mathrm{e} \mathrm{R}=$ Resolve (Capacidade resolutiva), que representa a satisfação do membro familiar com o tempo compartilhado entre eles. APGAR é composto por 5 questões, uma para cada letra citada, em que "sempre" vale 2 pontos, "algumas vezes" 1 ponto e "nunca" 0 pontos, o que somados resultarão em 0-4 elevada disfunção familiar, 5-6 moderada disfunção familiar e 7-0 boa funcionalidade família. Ao final do questionário, há 2 questões subjetivas: "com quem você mora?" e "você mora sozinho (a) por favor relacione abaixo as pessoas a quem você procura, mais frequentemente, quando precisa de ajuda", utilizadas para avaliar a funcionalidade da família nos pacientes da mostra.

Os resultados foram analisados no programa de análise estatística SPSS versão 24.0 e descritos na forma de frequência, média e desvio padrão, mediana (mínimo - e máximo). As respostas subjetivas foram analisadas qualitativamente e descritas na forma de frequência. A associação entre as variáveis foi analisada pela correlação de Pearson e foi considerado significativo um $p \leq 0,05$.

\section{RESULTADOS}

Foram incluídos no estudo 21 pacientes com DPOC, idade média de 66,3 \pm 10 anos, sendo 11 (52\%) sujeitos do sexo masculino, predominância de caucasianos $(n=19$; 90,4\%) e nível de escolaridade baixo $(n=13 ; 62,9 \%)$ (Tabela 1). Observou-se uma predominância de grau de parentesco muito próximo (cônjuge + filhos $=81 \%$ ), no núcleo familiar ao qual o paciente se referiu no APGAR da Família.

\begin{tabular}{|c|c|}
\hline Variáveis & Pacientes $(n=21)$ \\
\hline Idade (anos) & $66,3 \pm 10$ \\
\hline Sexo Feminino, n (\%) & $10(47,7)$ \\
\hline Sexo Masculino, n (\%) & $11(52,3)$ \\
\hline \multicolumn{2}{|l|}{ Raça } \\
\hline Caucasiano, n (\%) & $19(90,4)$ \\
\hline Pardo, n (\%) & $2(9,5)$ \\
\hline \multicolumn{2}{|l|}{ Escolaridade } \\
\hline Analfabeto & $1(4,8)$ \\
\hline Ensino Fundamental Incompleto & $8(39,1)$ \\
\hline Ensino Fundamental Completo & $4(19)$ \\
\hline Ensino Médio Incompleto & $1(4,8)$ \\
\hline Ensino Médio Completo & $5(23,8)$ \\
\hline Ensino Superior Incompleto & $1(4,8)$ \\
\hline Ensino Superior Completo & $1(4,8)$ \\
\hline \multicolumn{2}{|l|}{ Grau de parentesco, n (\%) } \\
\hline Cônjuge & $11(52)$ \\
\hline Filhos & $6(29)$ \\
\hline Outros & $4(19)$ \\
\hline
\end{tabular}

Dados expressos em média \pm desvio padrão; n: número amostral; (\%): frequência. 
O estadiamento da DPOC variou entre moderado e muito severo; no estado nutricional predominou a obesidade (IMC: $\mathrm{n}=10 ; 47,7 \%)$ e ex-tabagista $(\mathrm{n}=17$; $81 \%)$ com relação maços/anos 50,4 $\pm 31,7$. Entre os medicamentos utilizados, 4 (19\%) sujeitos relataram utilizar de forma regular antidepressivos e 3 (14\%) ansiolíticos conforme a prescrição médica (Tabela 2).

Tabela 2. Características clínicas dos pacientes avaliados.

\begin{tabular}{|c|c|}
\hline Variáveis & Pacientes $(\mathrm{n}=\mathbf{2 1})$ \\
\hline IMC, $\left(\mathrm{kg} / \mathrm{m}^{2}\right)$ & $29 \pm 7,8$ \\
\hline \multicolumn{2}{|l|}{ Classificação IMC, n (\%) } \\
\hline Magreza & $5(23,7)$ \\
\hline Eutrófico & $6(28,5)$ \\
\hline Obesidade & $10(47,7)$ \\
\hline \multicolumn{2}{|l|}{ Tabagismo, n (\%) } \\
\hline Ex-tabagista & $17(81)$ \\
\hline Tabagista & $4(19)$ \\
\hline № de cigarros fumados, (maços/anos) & $50,4 \pm 31,7$ \\
\hline \multicolumn{2}{|l|}{ Função pulmonar } \\
\hline $\mathrm{VEF}_{1},(\mathrm{I} / \mathrm{s})$ & $1,2 \pm 0,4$ \\
\hline $\mathrm{VEF}_{1}$, (\%predito) & $44,3 \pm 13,7$ \\
\hline $\mathrm{VEF}_{1} / \mathrm{CVF}$ & $53,1 \pm 13,2$ \\
\hline$V_{E F} / C V F(\%$ predito) & $66,6 \pm 17,3$ \\
\hline \multicolumn{2}{|l|}{ Estadiamento da DPOC, $n$ (\%) } \\
\hline Moderado & $7(33,4)$ \\
\hline Severo & $10(47,6)$ \\
\hline Muito severo & $4(19)$ \\
\hline \multicolumn{2}{|l|}{ Fármacos para afecções psicológicas, n (\%) } \\
\hline Antidepressivos & $4(19)$ \\
\hline Ansiolíticos & $3(14)$ \\
\hline Apgar da Família, (pontos) & $8 \pm 2,1$ \\
\hline \multicolumn{2}{|l|}{ Grau de disfunção, n (\%) } \\
\hline Boa funcionalidade familiar & $17(80,9)$ \\
\hline Moderada disfunção familiar & $2(9,5)$ \\
\hline Elevada disfunção familiar & $2(9,5)$ \\
\hline
\end{tabular}

Dados expressos em média \pm desvio padrão; n: número amostral; (\%): frequência; IMC: Índice de massa corporal, VEF; volume expiratório forçado no primeiro segundo; CVF: capacidade vital forçada; I/s: litros por segundo. 'GOLD, 2017 (15).

Foi constatado, através do APGAR de Família que 17 pacientes possuíam "boa funcionalidade familiar" e os demais pacientes relataram de moderada $(n=2)$ e elevada ( $\mathrm{n}=2$ ) “disfunção familiar" (Tabela 2). Dos que relataram moderada e elevada "disfunção familiar", os acrônimos mais comprometidos (0 pontos) foram "companheirismo", "afetividade" e "desenvolvimentos" (Figura 1).

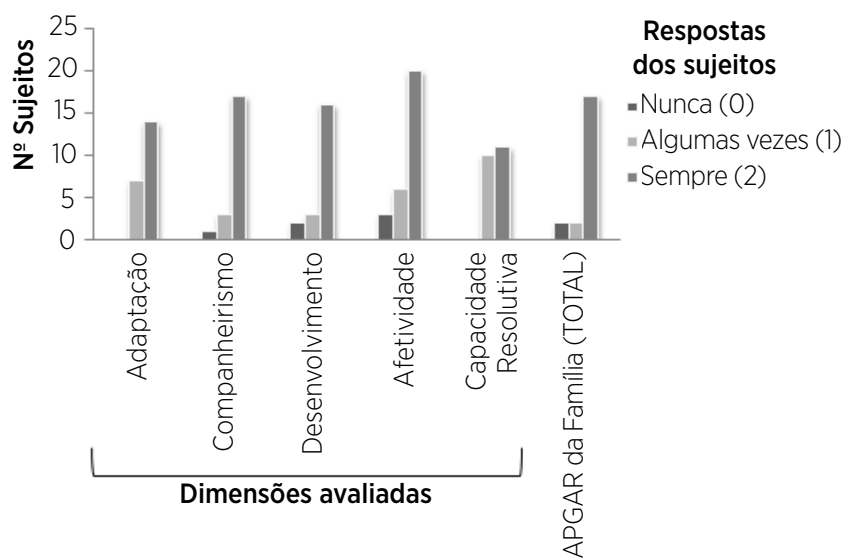

Figura 1. APGAR de Família. Representação gráfica dos Acrônimos (dimensões avaliadas) que compõem o APGAR de Família conforme a respostas dos pacientes ( $0=$ nunca; 1 = algumas vezes; 2 = sempre) e pontuação total. №= número de sujeitos.

Observamos uma correlação positiva entre o $\mathrm{VEF}_{1}$ (\%predito) e a pontuação do APGAR da Família apenas para os pacientes com "Boa funcionalidade familiar" ( $p=0,004 ; r=0,697)$, ou seja, para os indivíduos que apresentam menor grau de obstrução das vias aéreas são os que possuem melhor funcionalidade familiar (Figura 2).

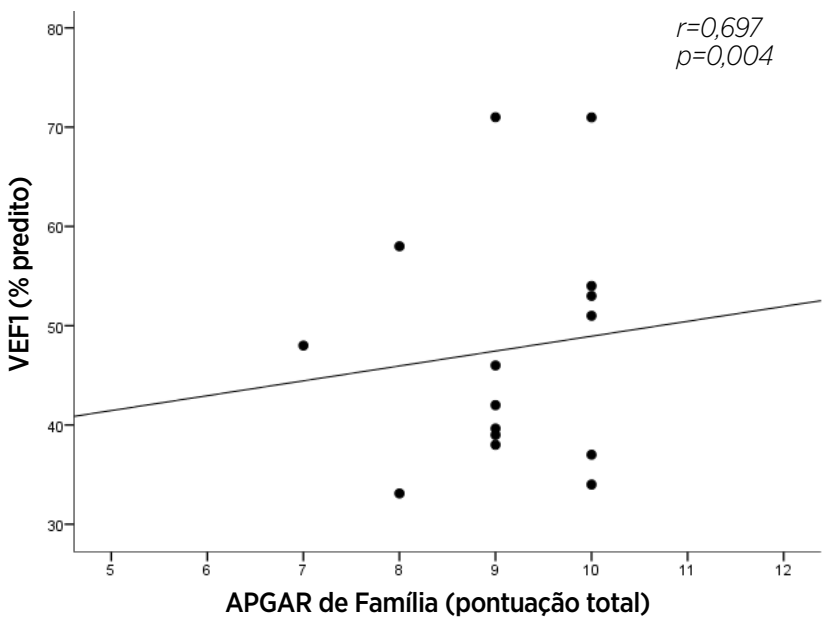

Figura 2. Associação entre $V_{E}$ e APGAR de Família em pacientes com "Boa funcionalidade familiar". Correlação entre o percentual do predito para o Volume Expiratório Forçado no 1 segundo (VEF\%predito) e pontuação do APGAR da Família, para os pacientes com "Boa funcionalidade familiar".

\section{DISCUSSÃO}

Os principais resultados encontrados no presente estudo são: (1) em grande maioria, os pacientes com DPOC que frequentam o Programa de Reabilitação Pulmonar apresentam boa funcionalidade familiar avaliada pelo APGAR da Família; (2) entre os pacientes que 
apresentam disfunção familiar, esta encontra-se bastante comprometida nas dimensões "companheirismo", "afetividade" e "desenvolvimentos"; e (3) o grau de obstrução das vias áreas associa-se positivamente à funcionalidade da família.

Estudos demonstraram que o instrumento APGAR de Família permitiu mensurar a compreensão da funcionalidade de familiares, de modo objetivo e sistematizado, relatado a partir da visão dos sujeitos com DPOC ${ }^{10,11}$. O APGAR de Família ofereceu resultados precisos e confiáveis, reconhecendo rapidamente qualquer disfunção familiar presente, bem como foi capaz de identificar as características (sexo e idade) e o grau de parentesco do familiar ${ }^{10,11}$.

Apesar de distribuição equivalente entre os sexos em nosso estudo, nenhuma mulher apresentou disfunção familiar; em contrapartida 4 homens apresentaram graus moderados e elevados de disfunção familiar. As menores pontuações encontradas em nosso estudo deram-se nos acrônimos "companheirismo" (satisfação pela forma como a família discute os assuntos de interesse comenta e compartilha a solução do problema), "desenvolvimento" (satisfação do membro familiar com a liberdade disponibilizada pela família para mudanças de papéis e para alcance de maturidade ou desenvolvimento emocional) e "afetividade" (satisfação do membro familiar com a intimidade e interações emocionais).

Nossos resultados vão ao encontro do estudo de Gomes e Pinheira ${ }^{12}$, em que os pacientes idosos com dor crônica também não estavam satisfeitos nas dimensões "companheirismo". No entanto, nesse estudo também foi encontrada menor pontuação na dimensão "capacidade resolutiva" (satisfação com o tempo que passam com a sua família) ${ }^{12}$. A carência de funcionalidade familiar acarreta muitas vezes em sintomas físicos, emocionais e sociais, o que, associado à doença crônica, pode ser ainda mais agravante, considerando-se as limitações de liberdade que ela pode causar, resultando no sentimento de solidão ${ }^{12}$.

Foi constatado que o nível de solidão em sujeitos com doenças crônicas, principalmente idosos, é mais elevado quando comparado aos sujeitos que não possuem estas doenças $^{12}$. A DPOC é uma doença que produz consequências significativas na vida de seus portadores, limitando-a em vários aspectos, o que afirma e reforça a questão da carência de liberdade, levando muitas vezes a sensação de abandono pela família e por pessoas próximas ${ }^{13}$.

A necessidade de suprir os sentimentos de afetividade, companheirismo e desenvolvimento é perceptível nos achados deste estudo, pois os 4 pacientes que relataram ter algum tipo de disfunção familiar são os mais assíduos no PRC, relatando o seu contentamento e bem-estar em participar do programa. Sujeitos com doenças crônicas associadas à idade avançada podem gerar grandes desafios, pois necessitam de apoio e cuidados diários ${ }^{11}$, estando a disfunção familiar diretamente ligada com a priorização de interesses que muitas vezes são apenas individuais, não comprometendo-se nem assumindo o papel que os compete como rede de apoio e culpando muitas vezes os familiares doentes ${ }^{12}$.

Desta forma, as implicações clínicas deste estudo denotam a importância da fisioterapia e do PRC na vida destes pacientes, pois além de aumentar a tolerância ao exercício, pode diminuir a chance de desenvolver depressão e ansiedade, promovendo uma melhor qualidade de vida em indivíduos com DPOC. Os benefícios na saúde física e mental podem ser relatados inclusive por familiares, com importante papel no controle e compreensão da doença ${ }^{14}$. Esta afirmativa corrobora com os nossos achados, em que a maioria dos pacientes que frequentam o PRC apresentam "boa funcionalidade da família" e alguns poucos pacientes consumiam fármacos como antidepressivos e ansiolíticos.

No que tange a associação entre a obstrução das vias aéreas e o APGAR de Família, este é um achado inédito do nosso estudo e, portanto, carece de literatura contemporânea para discussão do mesmo. $\mathrm{O}$ fato é que, comprovadamente, a DPOC gera um círculo vicioso entre obstrução das vias aéreas, dispneia, diminuição da capacidade funcional e isolamento social sucessivamente ${ }^{8}$. Com a progressão da doença, este quadro tende ao agravamento e maior dependência física, psíquica e emocional do paciente $\mathrm{DPOC}^{3}$. Desta forma, a própria fisiopatologia é capaz de explicar a correlação encontrada.

O presente estudo apresenta algumas limitações, como a não aplicação do Questionário Mini Exame de Estado Mental (MEEM) em todos os sujeitos da pesquisa; porém, ressaltamos que estes sujeitos participavam ativamente do PRC e as suas habilidades cognitivas estavam preservadas. No mesmo sentido, o fato de todos os sujeitos frequentarem um PRC pode ser um fator limitante que não permite extrapolar os resultados para aqueles indivíduos não realizam um PRC.

Pacientes acometidos por DPOC que frequentam o PRC apresentam em sua maioria uma boa funcionalidade familiar no seu cotidiano. Apesar deste contexto favorável, a gravidade da DPOC associou-se ao APGAR de Família e isto nos faz refletir que, com a progressão da doença, esta funcionalidade poderá deteriorar-se. Dos pacientes que apresentaram "disfunção familiar", estes relataram falta de "companheirismo", "afetividade" e "desenvolvimentos". 
Neste sentido, ressaltamos que, para além dos aspectos clínicos que um PRC pode melhorar, os aspectos psicológicos devem ser levados em consideração, bem como a rede de apoio que estes pacientes necessitam.

\section{AGRADECIMENTOS}

Ao Hospital Santa Cruz; Programa de Iniciação Científica (PUIC) e de Extensão (PROBEX) da Universidade de Santa Cruz do Sul; Programa de Iniciação Científica da Fundação de Amparo à Pesquisa do Estado do Rio Grande do Sul.

\section{REFERÊNCIAS}

1. Martinez CH, Mannino DM, Divo MJ. Defining COPD-Related Comorbidities, 2004-2014. Chronic Obstr Pulm Dis. 2014;1(1):5163. doi: 10.5216/ree.v16i1.22514

2. Tselebis A, Pachi A, llias I, Kosmas E, Bratis D, Moussas G et al. Strategies to improve anxiety and depression in patients with COPD: A mental health perspective. Neuropsychiatr Dis Treat. 2016;12:297-328. doi: 10.2147/NDT.S79354

3. Patel AR, Patel AR, Singh S, Singh S, Khawaja I. Global Initiative for Chronic Obstructive Lung Disease: The Changes Made. Cureus. 2019;11(6):e4985. doi: 10.7759/cureus.4985

4. Ministério da Saúde (BR). Perfil da morbimortalidade por doenças respiratórias crônicas no Brasil, 2003 a 2013. Bol Epidemiol. 2016;47(19):1-9.

5. Deng $X$, Song J. Correlation analysis between depression and family fitness in chronic obstructive pulmonary disease inpatients: A cross-sectional study. Med (Baltimore). 2018;97(52):e13946. doi: 10.1097/MD.0000000000013946.

6. Graham BL, Steenbruggen I, Barjaktarevic IZ, Cooper BG, Hall GL, Hallstrand S et al. Standardization of spirometry 2019 update an official American Thoracic Society and European Respiratory Society technical statement. Am J Respir Crit Care Med. 2019;200(8):E70-88. doi: 10.1164/rccm.201908-1590ST.

7. Raggi P. The never ending search for the elusive vulnerable plaque. Atherosclerosis. 2017;263(2006):311-2. doi: 10.1590/ s1806-37132007000400008

8. Global Initiative for Chronic Obstructive Lung Disease. GOLD Pocket Guide 2020 (COPD). Fontana-on-Geneva-Lake; 2020. Available from: www.goldcopd.org

9. Smilkstein $\mathrm{G}$. The family APGAR: a proposal for a family function test and its use by physicians. J Fam Pract. 1978;6(6):1231-9.

10. Vera I, Lucchese R, Nakatani AYK, Sadoyama G, Bachion MM, Vila VSC. Fatores associados à disfuncionalidade familiar em idosos não institucionalizados. Texto e Context Enferm. 2015;24(2):494-504. doi: 10.5216/ree.v16i1.22514.

11. Vera I, Lucchese R, Munari DB, Nakatani AYK. Índex APGAR de Família na avaliação de relações familiares do idoso: revisão integrativa. Rev Eletron Enferm. 2014;16(1):199-210. doi.org/10.1590/0104-07072015001602014

12. Gomes IS, Pinheira VMB. Dor crónica, funcionalidade familiar e solidão em pessoas idosas seguidas em consulta de dor. Rev Ibero-Am Saude Envelhec. 2018;4(1):1282-95. doi. org/10.24902/r.riase.2018.4\%281\%29.1281

13. Kerkoski E, Maria D, Vieira G, Chesani FH. A tristeza de viver com falta de ar : experiências de pessoas com doença pulmonar obstrutiva crônica. Proceedings of the $7^{\text {th }}$ Congresso Ibero-Americano em Investigação Qualitativa; 2018 Jul 10-13; Fortaleza, Brazil. Aveiro: Ludomedia; 2018. p. 753-62.

14. Marquese A, Jácome C, Cruz J, Gabriel R, Brooks D, Figueiredo D. Family-based psychosocial support and education as part of pulmonary rehabilitation in COPD: A randomized controlled trial. Chest. 2015;147(3):662-72. doi: 10.1378/chest.14-1488. 